\title{
ATRX, IDH1-R132H and Ki-67 immunohistochemistry as classification scheme for astrocytic tumors
}

\author{
Jinquan Cai ${ }^{1,6, *}$, Chuanbao Zhang ${ }^{2,3, *}$, Wei Zhang ${ }^{2,3}$, Guangzhi Wang ${ }^{1,6}$, Kun Yao ${ }^{6,7}$, \\ Zhiliang Wang ${ }^{3,6}$, Guanzhang Li ${ }^{2,6}$, Zenghui Qian ${ }^{2,6}$ Yongli Li ${ }^{1,6}$, Tao Jiang ${ }^{2,3,4,5,6}$, \\ Chuanlu Jiang ${ }^{1,6}$ \\ ${ }^{1}$ Department of Neurosurgery, The Second Affiliated Hospital of Harbin Medical University, NanGang District, Harbin, \\ Heilongjiang Province 150001, China \\ ${ }^{2}$ Beijing Neurosurgical Institute, Capital Medical University, Dongcheng District, Beijing 100050, China \\ ${ }^{3}$ Department of Neurosurgery, Beijing Tiantan Hospital, Capital Medical University, Dongcheng District, Beijing 100050, \\ China \\ ${ }^{4}$ Beijing Institute for Brain Disorders Brain Tumor Center, Dongcheng District, Beijing 100050, China \\ ${ }^{5}$ China National Clinical Research Center for Neurological Diseases, Dongcheng District, Beijing 100050, China \\ ${ }^{6}$ Chinese Glioma Cooperative Group (CGCG), Dongcheng District, Beijing 100050, China \\ ${ }^{7}$ Department of Pathology, Sanbo Brain Hospital, Capital Medical University, Haidian District, Beijing 100093, China \\ "These authors have contributed equally to the work
}

Correspondence to: Yongli Li, email: liyongli9999@aliyun.com Chuanlu Jiang, email: jcl6688@163.com

Tao Jiang, email: taojiang1964@163.com

Keywords: ATRX, IDH-R132H, Ki-67, astrocytic tumors, progression

Received: May 10, $2016 \quad$ Accepted: August 12, $2016 \quad$ Published: September 06, 2016

This is an open-access article distributed under the terms of the Creative Commons Attribution License, which permits unrestricted use, distribution, and reproduction in any medium, provided the original author and source are credited.

\section{ABSTRACT}

Recurrence and progression to higher grade lesions are key biological events and characteristic behaviors in the evolution process of glioma. Malignant astrocytic tumors such as glioblastoma (GBM) are the most lethal intracranial tumors. However, the clinical practicability and significance of molecular parameters for the diagnostic and prognostic prediction of astrocytic tumors is still limited. In this study, we detected ATRX, IDH1-R132H and Ki-67 by immunohistochemistry and observed the association of IDH1-R132H with ATRX and Ki-67 expression. There was a strong association between ATRX loss and IDH1-R132H $(p<0.0001)$. However, Ki-67 high expression restricted in the tumors with IDH1-R132H negative $(p=0.0129)$. Patients with IDH1-R132H positive or ATRX loss astrocytic tumors had a longer progressivefree survival $(p<0.0001, p=0.0044$, respectively). High $\mathrm{Ki}-67$ expression was associated with shorter PFS in patients with astrocytic tumors $(p=0.002)$. Then we characterized three prognostic subgroups of astrocytic tumors (referred to as A1, A2 and $A 3$ ). The new model demonstrated a remarkable separation of the progression interval in the three molecular subgroups and the distribution of patients' age in the A1-A2-A3 model was also significant different. This model will aid predicting the overall survival and progressive time of astrocytic tumors' patients.

\section{INTRODUCTION}

Astrocytic tumors are the most common group of human gliomas with inherent tendency for recurrence and malignant progression [1]. Malignant astrocytic tumors such as glioblastoma (GBM) are the most lethal intracranial tumors [2]. The clinical outcome of patients with astrocytic tumors depends on several factors, most notably age at diagnosis, clinical status as measured by the Karnofsky score, and tumor resection extent, as well 
as the histological classification, tumor grade and key molecular genetic alteration [3]. Despite the treatment of neurosurgical resection, radiotherapy and chemotherapy, most astrocytic tumors still grow continuously and tend to progress to a higher grade, leading to neurological disability and ultimately to death [4]. During recent years, large-scale research efforts - spearheaded by The Cancer Genome Atlas (TCGA) have made rapid advances in understanding glioma genetics. Isocitrate dehydrogenase $(I D H)$ mutations were detected in approximately $80 \%$ of diffuse and anaplastic astrocytomas as well as secondary GBMs [5]. The oncogenic IDH1 mutations mainly represented a change of guanine to adenine at position 395 (G395A), leading to the replacement of arginine by histidine at codon 132 (IDH1-R132H) at the enzymatic active site $[5,6]$. Patients with IDH1-R132H positive astrocytic tumors had a better outcome than those with IDH1-R132H negative astrocytic tumors. Recently, several teams demonstrated that $I D H$ mutations and ATRX status, combined with other classical biomarkers, refined the molecular classification of adult gliomas, providing a prognostic tool for clinicians [7-11]. These studies supported the development of a new molecular classification of IDH1-R132H and loss of ATRX, in that the clinical characteristics and prognosis of patients with grade II/III glioma and GBM are not accurately reflected by histological classifications [8, 9, 11-13]. The "Haarlem Consensus Guidelines for Nervous System Tumor Classification” [14] suggest that some entities will require molecular information to provide an "integrated" diagnosis, which is based on several layers comprising (i) the integrated diagnosis as top layer, followed by (ii) histological classification, (iii) WHO grade, and (iv) molecular information [14]. However, the clinical practicability and significance of molecular parameters for the diagnostic and prognostic prediction of astrocytic tumors is still limited. In this study, we detected ATRX, IDH1-R132H and Ki-67 by immunohistochemistry and characterized three prognostic subgroups of astrocytic tumors (referred to as A1, A2 and A3).

\section{RESULTS}

\section{Detection of ATRX, IDH1-R132H and Ki-67 in astrocytic tumors by immunohistochemistry}

In our dataset, ATRX nuclear protein, IDH1-R132H and $\mathrm{Ki}-67$ was detected by immunohistochemistry. IDH1$\mathrm{R} 132 \mathrm{H}$ dominated in diffuse astroctytoma (29/50, 58\%) and anaplastic astrocytoma $(5 / 9,55.6 \%)$ compared with in primary GBMs $(9 / 58,15.5 \%)$ (Figure 1A; Table 1, $\mathrm{p}<0.0001$, Chi-Square test). The frequency of ATRX loss was higher in As $(41 / 50,82 \%)$ and AAs $(7 / 9,77.8 \%)$ than that in pGBMs $(7 / 58,12.1 \%$ ) (Figure 1B; Table $1, \mathrm{p}<0.0001$, Chi-Square test). Primary GBMs (50/58, $86.2 \%$ ) expressed higher $\mathrm{Ki}-67$ protein than diffuse and anaplastic astrocytoma $(1 / 58,1.7 \%)$ (Figure 1C; Table 1, $\mathrm{p}<0.0001$, Chi-Square test).

\section{Association of IDH1-R132H with ATRX and Ki-67 expression}

In accord with previous reports, among the 41 tumor samples with IDH1-R132H positive, 34 lacked ATRX nuclear protein, while 53 samples of 74 astrocytic tumors with IDH1-R132H negative expressed ATRX, indicating a strong association between ATRX loss and IDH1-R132H
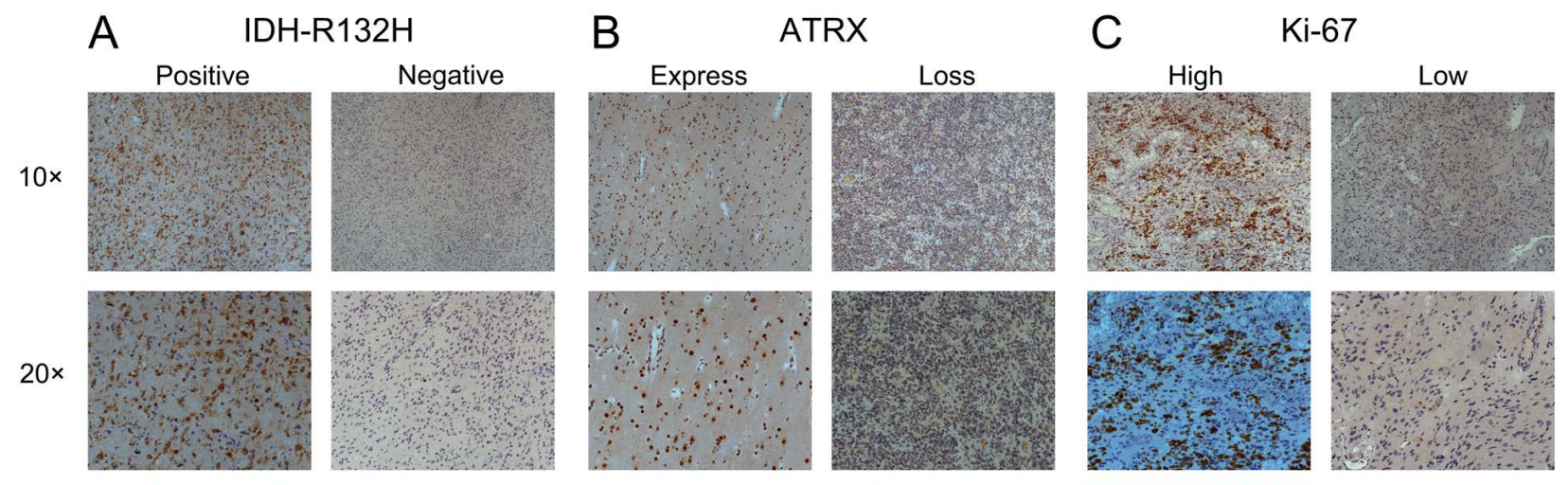

$40 \times$
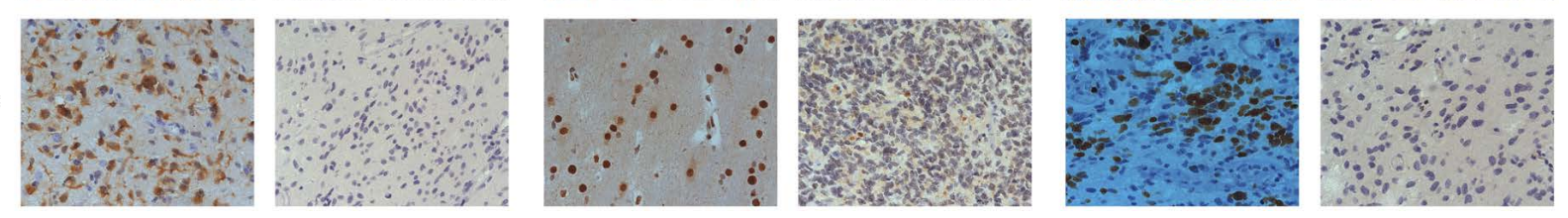

Figure 1: Detection of ATRX, IDH1-R132H and Ki-67 in astrocytic tumors by immunohistochemistry. A. IDH1-R132H positive or negative $(10 \times, 20 \times, 40 \times)$. B. ATRX nuclear protein expression or loss $(10 \times, 20 \times, 40 \times)$. C. Ki-67 high or low expression $(10 \times, 20 \times, 40 \times)$. 
Table 1: Clinicopathological characteristics of the patients in the cohort

\begin{tabular}{|c|c|c|c|c|c|}
\hline Variables & & $A(N=50)$ & $\mathbf{A A}(\mathbf{N}=9)$ & $\operatorname{GBM}(\mathrm{N}=58)$ & p value \\
\hline Median age (year) & & 38 & 39 & 44 & 0.0566 \\
\hline \multirow{2}{*}{ Age } & $\leq 42$ years old & 32 & 6 & 23 & \multirow{2}{*}{0.0273} \\
\hline & $>42$ years old & 18 & 3 & 35 & \\
\hline \multirow[b]{2}{*}{ Gender } & Male & 28 & 5 & 33 & \multirow[b]{2}{*}{0.9942} \\
\hline & Female & 22 & 4 & 25 & \\
\hline \multirow{2}{*}{ IDH1-R132H } & Positive & 29 & 5 & 9 & \multirow{2}{*}{$<0.0001$} \\
\hline & Negative & 21 & 4 & 49 & \\
\hline \multirow[b]{2}{*}{ ATRX-loss } & Yes & 41 & 7 & 7 & \multirow[b]{2}{*}{$<0.0001$} \\
\hline & No & 9 & 2 & 51 & \\
\hline \multirow{2}{*}{ Ki-67 Expression } & High & 1 & 0 & 50 & \multirow{2}{*}{$<0.0001$} \\
\hline & Low & 49 & 9 & 8 & \\
\hline
\end{tabular}

(Figure 2A; $<<0.0001$, Chi-Square test). In addition, the Ki-67 high expression restricted in the tumors with IDH1$\mathrm{R} 132 \mathrm{H}$ negative, indicating a group astrocytic tumors with high cell proliferative capacity (Figure $2 \mathrm{~B} ; \mathrm{p}=0.0129$ ).

\section{Prognostic value of ATRX, IDH1-R132H and Ki- 67 for patients with astrocytic tumors}

Then we evaluated the value of ATRX, IDH1$\mathrm{R} 132 \mathrm{H}$ and $\mathrm{Ki}-67$ for predicting the progression of astrocytic tumors. We observed that patients with IDH1-R132H positive astrocytic tumors had a longer progressive-free survival (PFS) than those with IDH1R132H negative astrocytic tumors (Figure 3A; Median PFS of IDH1-R132H positive $=802$ days, Median PFS of IDH1-R132H negative $=461.5$ days; $p<0.0001$ ). ATRX loss was also a favorable prognostic factor in patients with astrocytic tumors (Figure 3B; Median PFS of ATRX loss $=693$ days, Median PFS of ATRX expression=459 days; $p=0.0044$ ). High Ki-67 expression was associated with shorter PFS in patients with astrocytic tumors (Figure 3C; Median PFS of high Ki-67 expression=405 days, Median PFS of low Ki-67 expression $=459$ days; $\mathrm{p}=0.002$ ).

\section{Classification of astrocytic tumors defined by ATRX, IDH1-R132H and Ki-67}

Based on the above results and previous research, we incorporated IDH1-R132H, ATRX and Ki-67 status detected by IHC into A1-A2-A3 model. We classified astrocytic tumors into IDH1-R132H positive and IDH1$\mathrm{R} 132 \mathrm{H}$ negative tumors and then defined IDH1-R132H positive and ATRX loss tumors as A1, IDH1-R132H negative tumors with high Ki-67 expression as A3, and grouped IDH1-R132H positive with ATRX expression and IDH1-R132H negative tumors with low Ki-67 expression into $\mathrm{A} 2$. We observed that the distribution of patients' age in the A1-A2-A3 model was significant different (Figure $2 \mathrm{C}, \mathrm{p}=0.003$ ). As showed in Figure $3 \mathrm{D}$, survival analysis of the new classification also demonstrated a remarkable separation of the clinical course in the three molecular subgroups (log-rank test, $\mathrm{p}<0.0001)$. The A1 subgroup was correlated with a better clinical outcome (Figure 4, Median PFS $=828.5$ days). In contrast, the A3 subgroup was associated with a poorer clinical outcome (Median PFS $=405$ days). Correlation of the A2 subgroup with respect to clinical outcome fell between the A1 and A3 subgroups (Median $\mathrm{PFS}=526$ days). To study the influence of the three molecular markers (IDH1, ATRX and Ki-67) we used in our classification schema, multivariate Cox regression analyses were used for the adjustment of these factors (Table 2). We confirmed that IDH1-R132H status and $\mathrm{Ki}-67$ expression were the independent prognostic factors in this cohort and the new classification scheme was dependent on these three factors to predict survival value. Furthermore, upon incorporation of only our classification and the WHO grading scheme (Table 2 ), the prognostic value of our classification was still significant, independent of the WHO grades, and served as an addition to the latter. 
A

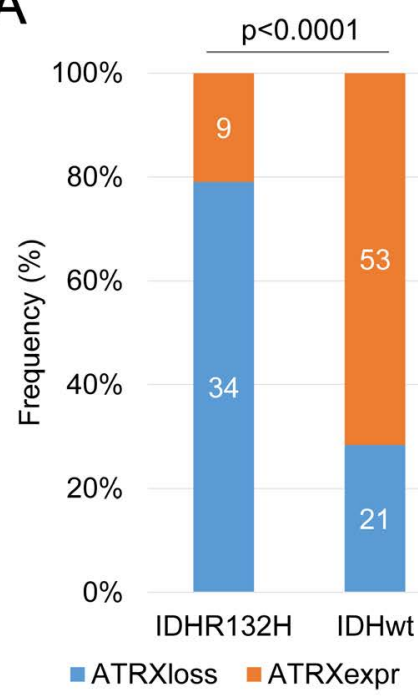

B

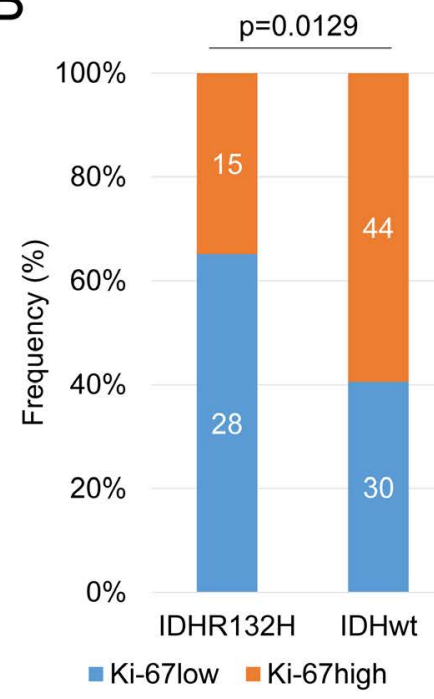

C

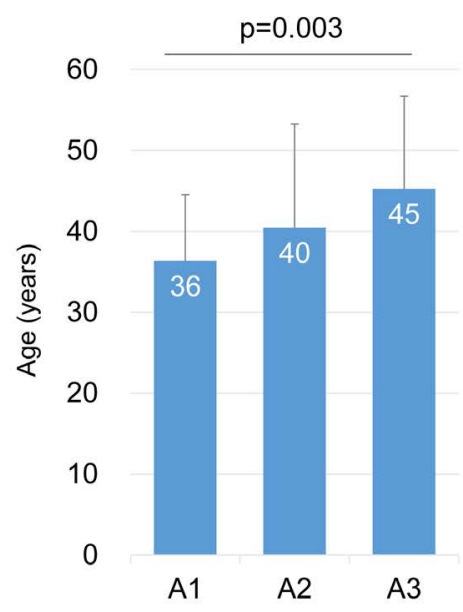

Figure 2: Correlation of ATRX and Ki-67 protein expression with IDH1-R132H status. A. There was a strong association between ATRX loss and IDH1-R132H ( $<00.0001)$. B. However, Ki-67 high expression restricted in the tumors with IDH1-R132H negative $(\mathrm{p}=0.0129)$. C. The distribution of patients' age in the A1-A2-A3 model was significant different $(\mathrm{p}=0.003)$.

A

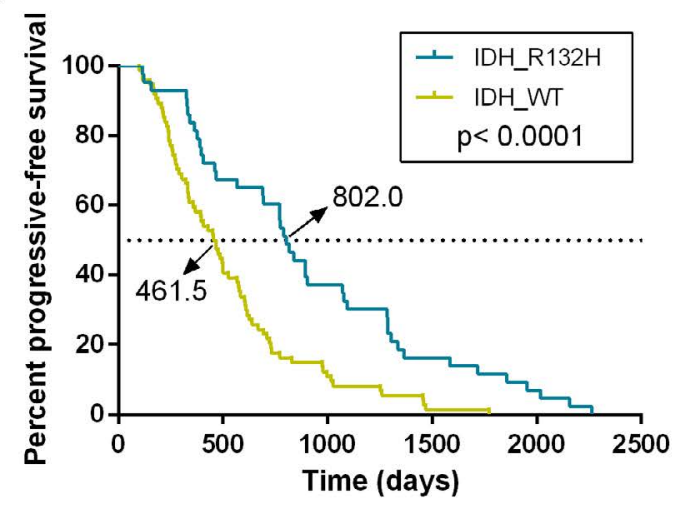

C

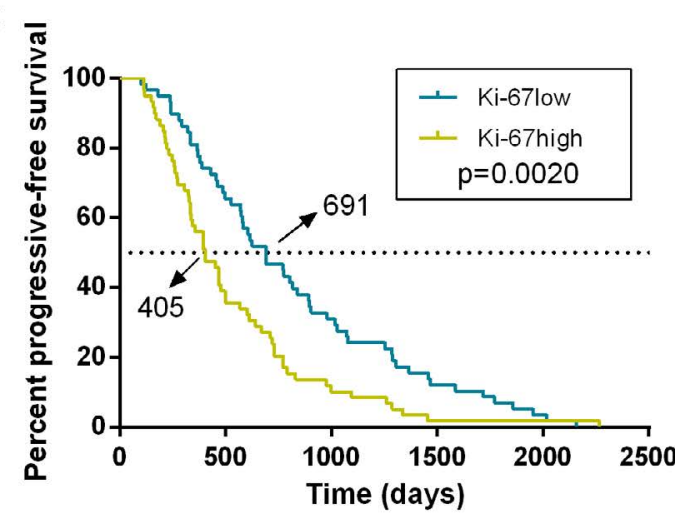

B

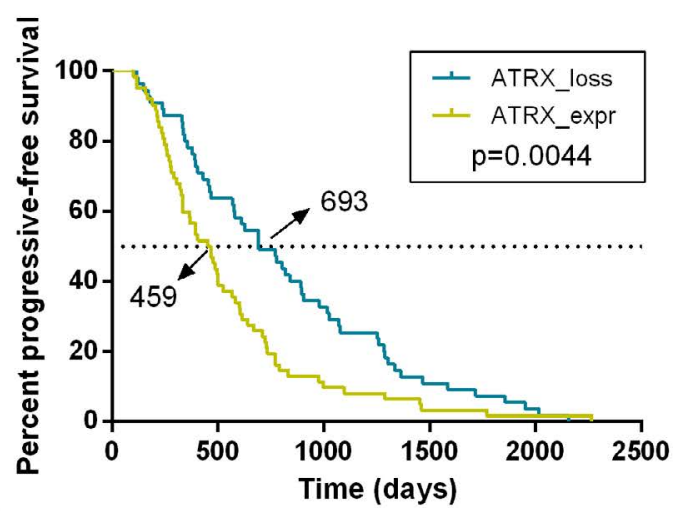

D

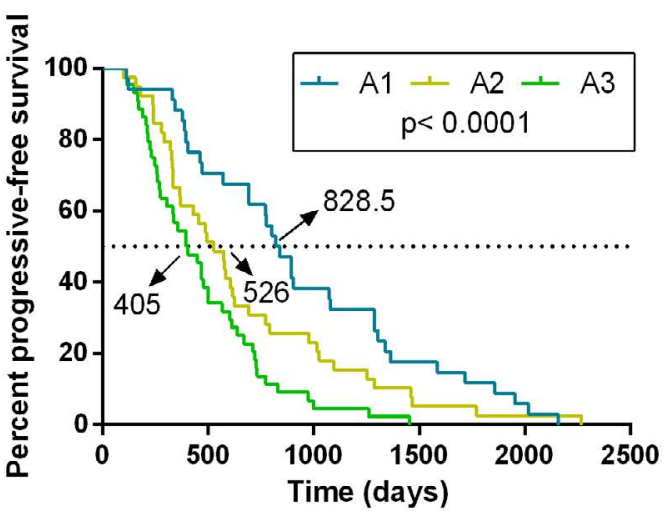

Figure 3: Kaplan-Meier estimates of survival for astrocytic tumor patients. A. Patients with IDH1-R132H positive astrocytic tumors had a longer progressive-free survival than those with IDH1-R132H negative astrocytic tumors $(\mathrm{p}<0.0001)$. B. ATRX loss was also a favorable prognostic factor in patients with astrocytic tumors $(\mathrm{p}=0.0044)$. C. High Ki-67 expression was associated with shorter PFS in patients with astrocytic tumors $(\mathrm{p}=0.002)$. D. Survival analysis of the new classification revealed a remarkable separation of the clinical course in the three molecular subgroups ( $\mathrm{p}<0.0001)$. 


\section{DISCUSSION}

Over the past decade, insights into the molecular pathology of gliomas have significantly improved both our biological understanding of neoplasms as well as our abilities to diagnose tumors and estimate their prognosis and likelihood of response to specific therapies [15]. In the present study, we detected the IDH1-R132H, ATRX and Ki-67 expression using immunohistochemistry, promoting the molecular neuropathology research to the clinical translational practice.

Nowadays, mutations in IDHI are commonly established as a hallmark molecular feature of grade II/III gliomas and secondary GBM which have predominant localization in the frontal and temporal lobes [15]. IDH1-R132H (G395A) is the most common mutation $(\sim 90 \%)$, followed at a distance by IDH1R132S (C394A), IDH1-R132C (C394T), IDH1-R132G (C394G), IDH1-R132L (G395T) and IDH1-R132V (C394G G395T) (0.5-5\%) [12]. Thus, IDH1-R132H can be used for the diagnosis between grade II/III gliomas, secondary GBM and primary GBM [16]. Mutations in the $I D H$ genes are thought to cause glioma-CpG island methylator phenotype (G-CIMP) within the proneural GBM subgroup [17]. IDH mutations seem to require cooperating mutations in ATRX, and they are less frequently detected in primary GBMs [5]. Mutations of ATRX inactivated the gene product and caused a lack of ATRX immunolabeling [18]. ATRX loss occurs almost exclusively in IDH mutant astrocytic tumors, and ATRX loss and $1 \mathrm{p} / 19 \mathrm{q}$ codeletion are largely mutually exclusive [19]. ATRX loss is characteristic in the refinement of the diagnosis of IDH mutant astrocytomas. Assessment of ATRX loss by immunohistochemical staining captures the majority of mutations, indicating that the use of immunohistochemical testing in routine neuropathology diagnostics gives a reasonable sensitivity [20].

In addition, our result showed that higher Ki67 expression mostly dominated in the IDH1-R132H negative cluster. Previously, our research delineated that IDH-wt/TERTp-mut gliomas expressed higher Ki67 protein and showed the evidence of cell proliferation. "Classical" gene expression was mostly restricted to the IDH-wt/TERTp-mut gliomas with the poorest survival. Now, we used negative IDH1-R132H combined with higher Ki-67 expression to define the cluster similar to the IDH-wt/TERTp-mut gliomas. In contrast with $I D H$ mutations and ATRX loss being widely considered as key aberrations in the early stage of astrocytic tumors, higher Ki-67 expression may be the final event in the progression of these tumors. We speculated that IDH1R132H accompanied by ATRX or Ki-67 may represent a distinct biological process during the development of astrocytic tumors from the original tumor cells.

Based on the above results and previous research, we incorporated IDH1-R132H, ATRX and Ki-67 status detected by IHC into A1-A2-A3 model. The new classification also demonstrated a remarkable separation of the progression interval in the three molecular subgroups and the distribution of patients' age in the A1-A2-A3 model was also significant different. This model will aid predicting the overall survival and progressive time of astrocytic tumors' patients.

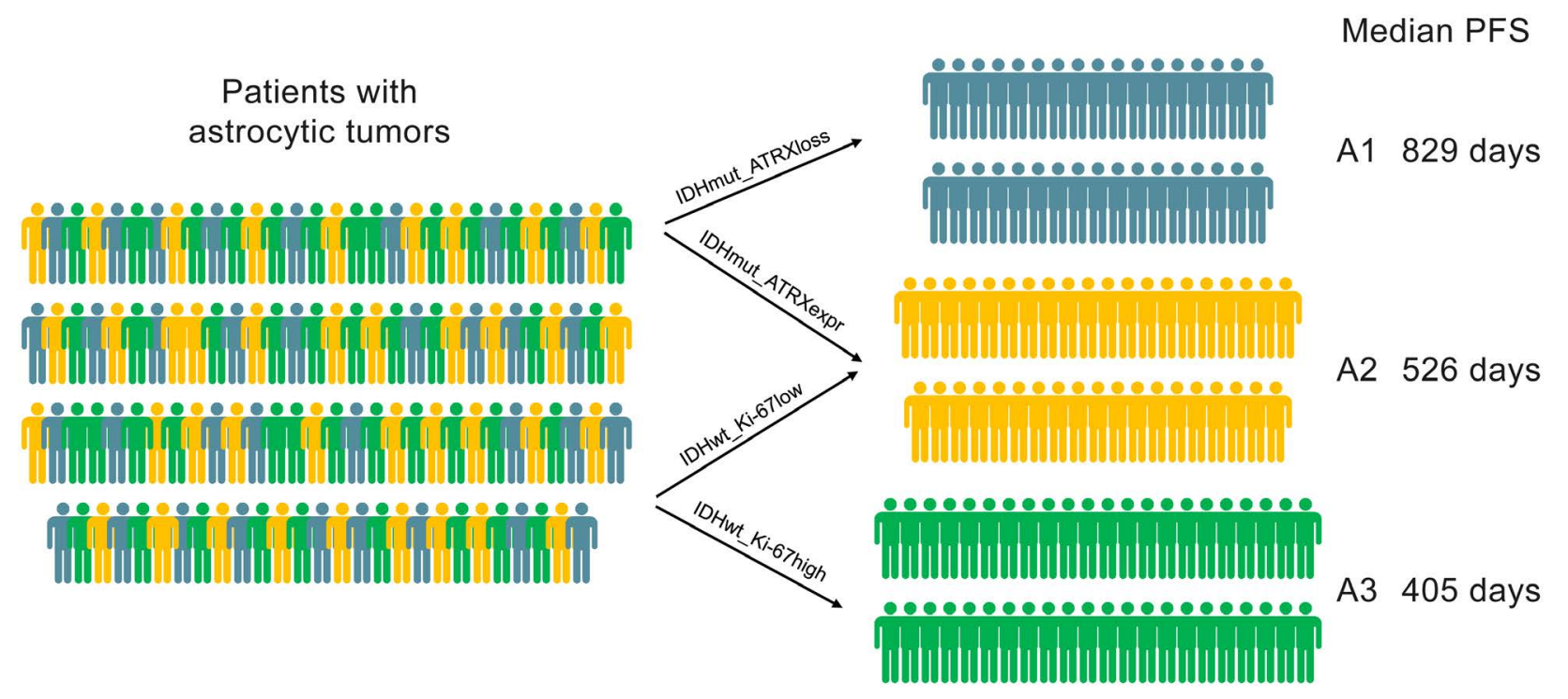

Figure 4: A1-A2-A3 Model for classification of astrocytic tumors based on ATRX, Ki-67 and IDH1-R132H status. IDH1-R132H positive tumors with ATRX loss were defined as A1 (median PFS=829 days), IDH1-R132H negative tumors with high Ki-67 expression as A3 (median PFS=526 days), and IDH1-R132H positive tumors with ATRX expression and IDH1-R132H negative tumors with low Ki-67 expression were termed as A2 (median PFS=405 days). 
Table 2: Cox regression analysis for the progression-free survival of patients with astrocytic Tumors

\begin{tabular}{|c|c|c|c|c|c|c|}
\hline \multirow{2}{*}{ Variable } & \multicolumn{3}{|c|}{ Univariate Cox Model } & \multicolumn{3}{|c|}{ Multivariate Cox Model } \\
\hline & HR & $95 \% \mathrm{CI}$ & p value & HR & $95 \% \mathrm{CI}$ & p value \\
\hline \multicolumn{7}{|c|}{ COX model of Classification, IDH, ATRX and Ki-67 } \\
\hline $\begin{array}{l}\text { Age } \leq 42 \text { vs. } \\
>42\end{array}$ & 1.366 & $0.944-1.976$ & 0.098 & & & \\
\hline Male vs. Female & 0.924 & $0.635-1.346$ & 0.682 & & & \\
\hline $\begin{array}{l}\text { IDH1-R132H } \\
\text { positive vs. } \\
\text { negative }\end{array}$ & 0.432 & $0.288-0.648$ & 4.97E-05 & 0.218 & $0.067-0.71$ & 0.012 \\
\hline $\begin{array}{l}\text { ATRX loss vs. } \\
\text { expression }\end{array}$ & 0.586 & $0.404-0.851$ & $5.00 \mathrm{E}-03$ & 0.941 & $0.559-1.585$ & 0.82 \\
\hline $\begin{array}{l}\text { Ki- } 67 \text { expression } \\
\text { low vs. high }\end{array}$ & 1.787 & $1.231-2.596$ & 0.002 & 2,738 & $1.139-6.581$ & 0.024 \\
\hline Classification & 1.672 & $1.316-2.126$ & 2.63E-05 & 0.5 & $0.188-1.330$ & 0.165 \\
\hline \multicolumn{7}{|c|}{ COX model of Classification and Grade } \\
\hline Classification & 1.672 & $1.316-2.126$ & $2.63 \mathrm{E}-05$ & 1.512 & $1.062-2.153$ & 0.022 \\
\hline $\begin{array}{l}\text { WHO grade II/ } \\
\text { III vs. IV }\end{array}$ & 1.449 & $1.187-1.770$ & 2.70E-04 & 1.121 & $0.835-1.504$ & 0.447 \\
\hline
\end{tabular}

\section{MATERIALS AND METHODS}

\section{Patients enrollment}

As a part of the Chinese Glioma Genome Atlas (CGGA) project (http://www.cgga.org.cn/portal.php), we consented patients who underwent surgical resection for malignant gliomas at the Glioma Treatment Center of Beijing Tiantan Hospital from January 2008 through March 2015. The study was approved by the ethics committee in both hospitals and written informed consent was obtained from each patient. All of data and samples were collected under the IRB of Beijing Tiantan Hospital. The criteria of enrollment include: age more than 18 years-old, histologically confirmed astrocytic tumors, relapse detected by MRI and patient's consent. 117 samples came into the cohort, containing astrocytoma (A, grade II), anaplastic astrocytoma (AA, grade III) and primary glioblastoma (GBM, grade IV). The histological diagnoses were confirmed by two neuropathologists according to the 2007 World Health Organization (WHO) classification guidelines. Specimens were collected after definitive diagnosis and stored as paraffin embedded blocks for subsequent molecular characterization. The collected specimens were verified by our pathologists to harbor $>80 \%$ viable tumor tissue. For each enrolled patient, patients' progression-free survival data were recorded when the relapse occurred.

\section{Immunohistochemistry for IDH1-R132H, ATRX and $\mathrm{Ki}-67$}

Immunostaining was performed according to the manufacturer's protocol. In brief, formalin-fixed, paraffinembedded tissue sections cut to four micrometer were dried at $80{ }^{\circ} \mathrm{C}$ for $15 \mathrm{~min}$ and dewaxed in xylene, rinsed in graded ethanol, and rehydrated in double-distilled water. The sections were then treated with $3 \% \mathrm{H}_{2} \mathrm{O}_{2}$ for 5 min at room temperature (RT) to block endogenous peroxidase activity. For antigen retrieval, slides were pretreated by steaming in sodium citrate buffer (10 $\mathrm{mM}$ sodium citrate, $\mathrm{pH} 6.0$ ) for $15 \mathrm{~min}$ at $100^{\circ} \mathrm{C}$. After washing with phosphate-buffered saline for $3 \mathrm{~min}$, the sections were immunostained with an anti-human IDH1-R132H antibody (at 1:60 dilution, H09, Dianova, Hamburg, Germany) or an anti-human ATRX antibody (at 1:800 dilution, ab97508, Abcam) or an anti-human Ki-67 protein antibody (Santa Cruz Biotechnology, Santa Cruz, CA), and incubated at $4{ }^{\circ} \mathrm{C}$ over night. After washed by 3 changes of PBS buffer, the tissues were covered by anti-mouse/rabbit polymer HRP-label for 30min at RT. Staining reaction was performed through covering tissue by prepared $\mathrm{DAB}$ chromogen solution, and incubating approximately for $10 \mathrm{~min}$ to allow for proper brown color development. Each slide was individually reviewed and scored by two experienced neuropathologists.

Standard of IDH1-R312H staining: (1) a strong cytoplasmic immunoreaction product was scored positive; 
(2) a weak diffuse staining and staining of macrophages were not scored positive [21,22].

Standard of ATRX staining according to German Cancer Research Center (DKFZ): nuclear ATRX loss was scored as specific if tumor cell nuclei were unstained while nuclei of non-neoplastic cells such as endothelia, microglia, lymphocytes and reactive astrocytes were strongly positive [12].

Staining was scored using a two-grade scale, with $0=$ no or $<10 \%$ occurrence of staining, $1=>10 \%$ of cells positively stained. Score 1 was defined as high Ki-67 expression [5].

\section{Statistical analysis}

Receiver operating characteristic (ROC) curves were constructed to determine the discriminatory capacity of IDH1-R132H and (or) ATRX loss for diagnosis. KaplanMeier analysis was performed to estimate the survival time of different subgroups and a log-rank test was used to test prognostic differences. Comparisons of binary and categorical patient characteristics between subgroups were performed by the use of the Fisher's exact test All statistical computations were performed with the statistical software environment $\mathrm{R}$ version 3.2.0 (http://www.r-project.org/), GraphPad Prism Version 6.01 or Microsoft Excel 2013. P value $<0.05$ was considered statistically significant.

\section{ACKNOWLEDGMENT}

We thank Yuling Yang for tissue sample collection and clinical data retrieval. This work was supported by grants from The Research Special Fund For Public Welfare Industry of Heath (No. 201402008), The National Key Research and Development Plan (No. 2016YFC0902500). National Key Technology Research and Development Program of the Ministry of Science and Technology of China (No. 2014BAI04B02), National High Technology Research and Development Program (No.2012AA02A508), Beijing Science and Technology Plan (No. Z131100006113018), International Science and Technology Cooperation Program (No. 2012DFA30470), National Natural Science Foundation of China (No.81372700,No. 81201993), Special Fund Project of Translational Medicine in the Chinese-Russian Medical Research Center (No. CR201417), Research Project of Chinese Society of Neurooncology, CACA (CSNO-2014-MSD08).

\section{CONFLICTS OF INTEREST}

None of the authors has a conflicts of interest regarding this study.

\section{REFERENCES}

1. Cai J, Chen J, Zhang W, Yang P, Zhang C, Li M, Yao K, Wang H, Li Q, Jiang C, Jiang T. Loss of ATRX, associated with DNA methylation pattern of chromosome end, impacted biological behaviors of astrocytic tumors. Oncotarget. 2015; 6:18105-18115.

2. Yang P, Zhang W, Wang Y, Peng X, Chen B, Qiu X, Li G, Li S, Wu C, Yao K, Li W, Yan W, Li J, You Y, Chen CC, Jiang T. IDH mutation and MGMT promoter methylation in glioblastoma: results of a prospective registry. Oncotarget. 2015; 6:40896-40906.

3. Stupp R, Brada M, van den Bent MJ, Tonn JC, Pentheroudakis G. High-grade glioma: ESMO Clinical Practice Guidelines for diagnosis, treatment and followup. Annals of oncology : official journal of the European Society for Medical Oncology / ESMO. 2014; 25 Suppl 3:iii93-101.

4. Chaichana KL, McGirt MJ, Laterra J, Olivi A, QuinonesHinojosa A. Recurrence and malignant degeneration after resection of adult hemispheric low-grade gliomas. Journal of neurosurgery. 2010; 112:10-17.

5. Cai J, Yang P, Zhang C, Zhang W, Liu Y, Bao Z, Liu X, Du W, Wang H, Jiang T, Jiang C. ATRX mRNA expression combined with IDH1/2 mutational status and Ki-67 expression refines the molecular classification of astrocytic tumors: evidence from the whole transcriptome sequencing of 169 samples samples. Oncotarget. 2014; 5:2551-2561.

6. Turkalp Z, Karamchandani J, Das S. IDH Mutation in Glioma: New Insights and Promises for the Future. JAMA neurology. 2014.

7. Bai H, Harmanci AS, Erson-Omay EZ, Li J, Coskun S, Simon M, Krischek B, Ozduman K, Omay SB, Sorensen EA, Turcan S, Bakirciglu M, Carrion-Grant G, Murray PB, Clark VE, Ercan-Sencicek AG, et al. Integrated genomic characterization of IDH1-mutant glioma malignant progression. Nat Genet. 2015.

8. Cancer Genome Atlas Research N. Comprehensive, Integrative Genomic Analysis of Diffuse Lower-Grade Gliomas. The New England journal of medicine. 2015.

9. Eckel-Passow JE, Lachance DH, Molinaro AM, Walsh KM, Decker PA, Sicotte H, Pekmezci M, Rice T, Kosel ML, Smirnov IV, Sarkar G, Caron AA, Kollmeyer TM, Praska $\mathrm{CE}$, Chada AR, Halder C, et al. Glioma Groups Based on 1p/19q, IDH, and TERT Promoter Mutations in Tumors. The New England journal of medicine. 2015.

10. Kim J, Lee IH, Cho HJ, Park CK, Jung YS, Kim Y, Nam SH, Kim BS, Johnson MD, Kong DS, Seol HJ, Lee JI, Joo KM, Yoon Y, Park WY, Lee J, et al. Spatiotemporal Evolution of the Primary Glioblastoma Genome. Cancer Cell. 2015; 28:318-328.

11. Suzuki H, Aoki K, Chiba K, Sato Y, Shiozawa Y, Shiraishi Y, Shimamura T, Niida A, Motomura K, Ohka F, Yamamoto T, Tanahashi K, Ranjit M, Wakabayashi T, Yoshizato T, Kataoka K, et al. Mutational landscape and clonal architecture in grade II and III gliomas. Nat Genet. 2015.

12. Reuss DE, Sahm F, Schrimpf D, Wiestler B, Capper D, Koelsche C, Schweizer L, Korshunov A, Jones DT, 
Hovestadt V, Mittelbronn M, Schittenhelm J, HeroldMende C, Unterberg A, Platten M, Weller M, et al. ATRX and IDH1-R132H immunohistochemistry with subsequent copy number analysis and IDH sequencing as a basis for an "integrated" diagnostic approach for adult astrocytoma, oligodendroglioma and glioblastoma. Acta Neuropathol. 2015; 129:133-146.

13. Leeper HE, Caron AA, Decker PA, Jenkins RB, Lachance $\mathrm{DH}$, Giannini C. IDH mutation, $1 \mathrm{p} 19 \mathrm{q}$ codeletion and ATRX loss in WHO grade II gliomas. Oncotarget. 2015.

14. Louis DN, Perry A, Burger P, Ellison DW, Reifenberger G, von Deimling A, Aldape K, Brat D, Collins VP, Eberhart C, Figarella-Branger D, Fuller GN, Giangaspero F, Giannini C, Hawkins C, Kleihues P, et al. International Society Of Neuropathology--Haarlem consensus guidelines for nervous system tumor classification and grading. Brain pathology (Zurich, Switzerland). 2014; 24:429-435.

15. Liu C-Y, Li Q-J, Cai J-Q. Evolving Molecular Genetics of Glioblastoma. Chinese medical journal. 2016; 129:464.

16. Kickingereder P, Sahm F, Radbruch A, Wick W, Heiland S, Deimling A, Bendszus M, Wiestler B. IDH mutation status is associated with a distinct hypoxia/angiogenesis transcriptome signature which is non-invasively predictable with rCBV imaging in human glioma. Scientific reports. 2015; 5:16238.

17. Lu C, Ward PS, Kapoor GS, Rohle D, Turcan S, AbdelWahab O, Edwards CR, Khanin R, Figueroa ME, Melnick
A, Wellen KE, O'Rourke DM, Berger SL, Chan TA, Levine RL, Mellinghoff IK, et al. IDH mutation impairs histone demethylation and results in a block to cell differentiation. Nature. 2012; 483:474-478.

18. Heaphy CM, de Wilde RF, Jiao Y, Klein AP, Edil BH, Shi C, Bettegowda C, Rodriguez FJ, Eberhart CG, Hebbar S, Offerhaus GJ, McLendon R, Rasheed BA, He Y, Yan H, Bigner DD, et al. Altered telomeres in tumors with ATRX and DAXX mutations. Science. 2011; 333:425.

19. Wiestler B, Capper D, Holland-Letz T, Korshunov A, von Deimling A, Pfister SM, Platten M, Weller M, Wick W. ATRX loss refines the classification of anaplastic gliomas and identifies a subgroup of IDH mutant astrocytic tumors with better prognosis. Acta Neuropathol. 2013; 126:443-451.

20. Brandner S, von Deimling A. Review: Diagnostic, prognostic and predictive relevance of molecular markers in gliomas. Neuropathology and applied neurobiology. 2015.

21. Capper D, Weissert S, Balss J, Habel A, Meyer J, Jager D, Ackermann U, Tessmer C, Korshunov A, Zentgraf H, Hartmann C, von Deimling A. Characterization of R132H mutation-specific IDH1 antibody binding in brain tumors. Brain pathology (Zurich, Switzerland). 2010; 20:245-254.

22. Cai, J., et al. (2016). "Detection of ATRX and IDH1$\mathrm{R} 132 \mathrm{H}$ immunohistochemistry in the progression of 211 paired gliomas." Oncotarget 7:16384-16395. 